Yitteilung aus der medizinischen Abteilung des chemischen Universitătslaboratoriums zu Freiburg i. B.

\title{
Ueber Ameisensäureausscheidung beim Menschen nach Einnahme von Methylalkohol, Hexamethyien- tetramin, ameisensaurem und milchsaurem Natrium sowie Traubenzucker.
}

\author{
Von Wi. Anteurieth.
}

Nach älteren Untersuchungen ( $r$. J a k s c h $h^{1}$ ) und $r . R$ ok i :

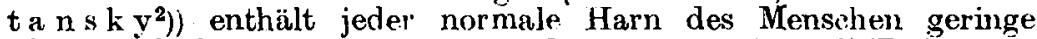
Mengen flüchtiger Fettsäureu, und zwar vorwiegend Essigsäure, neben wenig Ameisensäure und wahrscheinlich auch Buttersäure. Bei ausschließlicher Frnährung mit Mehlspeisen. also vorzugsweise mit Kohlenhydraten, sah $r$. R o $k$ i $t a n s k y$ die Tagesmenge der ausgeschiedenen flüchtigen Fettsiumren stark vermehrt. In den meisten Fällen wurde bisher die $G$ e s a $\mathrm{m} \mathrm{t} s$ u $\mathrm{m}$ m e der flüchtigen Fettsäuren cles Harns bestimmt, also ohne Berücksichtigung der einzelnen Bestandteile derselben. So haben $H$. Stra a $\beta$ und H. $P h$ ili p p s oh $n^{3}$, bei einer bestimmten Diät als Tagesmenge an flüchtigen Fettsäuren eine Menge gefnnden, die 40 bis $60 \mathrm{ccm}$ einer 1/10-Normal-Süur elösung entsprach. Beim Diabetes mellitus wurden für die flüchtigen Säuren des Harns zum Teil außerordentlich hohe "Säurezahlen" ermittelt. Ebenso bei gewiss'n Frkrankungen der Leber.

Ceber las Vorkommen von Ame isensianre im Harn des Mensehen liegen nur spärliche Angaben vor; nach älteren Anyaben ( $\mathrm{B} \cup 1 \mathrm{ig} \mathrm{i} n \mathrm{sk} \mathrm{v^{4 }}$ ) soll jecler Harn diese Säure in geringer Menge enthalfen: etwis größere Mengen sind von Salkow skib) bei Lenkäimid beobachtet worden. In normalen Hundeharn hat $\mathrm{P} \circ \mathrm{h} \mathrm{l}^{6}$ ) stets kleine Mengen von Ameisensäure nachweisen können. Von den als Natriumsalze eingeführten Fettsiuren bewirken nach s' ch ot $t\left(n^{7}\right)$ nur die Essigsiiure und in noch höherem Grade die Ameisensäure eine V'ermehrung der flüchtigen Fettsïuren des Harns; wenigstens hat dies Sch otten für Hunde nachgewiesen, in deren Organismus sich Essigsïure und Ameisensiiure als weit beständiger erwiesen haben als dic knhlenstoffreicheren Fettsäuren, denn nach Verfütterung von je $10--20 \mathrm{~g}$ von Capropsäure, Isovaleriansïure mul der beiden Buttersiuren in Form jhrer Natrium.

1) Zeitschr. f. phvsiol. (hent. 10. 536 (1886).

2) Wiener zued. Jahrbïches (2), 2, 206 (1887).

3) Zeitschr. it. klinische Medizin 40. 369 (1900).

4) Hoppe-Seyler's ,. Tintersuchmgen " 1866.240.

$\left.{ }^{5}\right)$ Zeitschr. f. physiol. Chemio 13. 264 (1889).

5) Archiv t'. experim. Pathologie u. Pharmakol. 31. 286 (1893).

i) Zeitschr. f. physinl. ('hem. 7. 375 (1882/83). 
salze war die Gesamtmenge der flüchtigen Fettsäuren im Harn der Hunde nur ganz wenig vermehrt, während eine erheblichere Vermehrung nach Verfütterung von essig- und ameisensaurem Natrium zu beobachten war. Diese Versuche zeigten aber auch andererseits, claß Ameisensäure im Körper des Hundes in größerem Umfange verbrannt wird. J. Pohl (l. c.) fand, ebenfalls beim Hunde, bei zwei Versuchen das eine Mal 5,8, clas andere Mal 18\% des eingeführten Formiates wieder und zwar in den innerhalb von drei Tagen gelassenen Harne der Versuchstiere.

Wïhrend der Harn nach Verfütterung der Natriumsalze der h $\ddot{b}$ h ep en Fettsäuren stark alkalisch war und mit Säuren lebhaft aufbrauste, war dies nach Fütterung von Natriumacetat und Natriumformiat lange nicht in dem Grade der Fall. (S c h o t t e n.) Ueber die Ausscheidung der Ameisensäure beim M ens ch e n nach Einnahme von ameisensauren Salzen und anderer Stoffe habe ich in der Literatur keine näheren Angaben vorgefunden.

Ueber das Sehicksal des Methylalkohols im tierischen Organismus hat $J$. Poh $l$ wohl als erster amfassende Versuche angestellt, und dieser Forscher hat auch die Giftwirkung les Methylalkohols im Vergleiche zu der des Aethylalkohols einem eingehenden Studium unterworfen. Nach $\mathbf{P}$ oh 1 geht an Hunden verfütterter Methylalkohol als solcher nicht in den Harn der Versuchstiere über; dagegen erfährt das unter Phosphorsäurezusatz aus dem Harn gewonnene Destillat der Norm gegenüber eine beträchtliche Zunahme der Acidität. Die Versuche beim Hund ließen eine länger andauernde, sich allmählich steigernde, damn aber wieder abnehmende Ameisensäureausscheidung deutlich erkennen. Erst am vierten Tage nach der Methylalkoholaufnahme war das Maximum der Ameisensäureausstheidung erreicht. Die Versuche von $P_{0}$ h 1 lehren, daß die Einführung des Methylalkohols in den Magen beim Hunde zu einer vermehrten Ausscheidung der Ameisensäure führt. Von einer Anfspeicherung des ameisensauren Salzes im Körper des Hundes kann nach Pohl nicht die Rede sein; die verlangsamte Ausscheidung der Ameisensäure durch die Nieren muß vielmehr darauf beruhen, daß der Methylalkohol als solcher oder als ein Derivat desselben im Tierkörper zunächst zurückgehalten, daselbst nur allmählich oxydiert und schließlich, wenigstens zum Teil, als A,meisensäure ausgeschieden wird. Pohl bestimmte den Oxydationsverlauf auch bei intravenöser Darreichung des Methylalkohols: auch bei dieser direkten Einfuhr ins Blut, gewissermaßen bei Ueberschwemmung des Organismus mit Methylalkohol, tritt an dem Oxydationsvorgange, insbesondere an den Typus der Ameisensäureausscheidung keine Aenderung gegenüber der Ausscheidung nach Aufnahme des Methylalkohols vom Magen aus ein. Bei dem vergleichenden Studium der physiologischen Wirkung des Methylalkohols und Formaldehyds zeigte sich, daB bei keiner Applikationsart des Formaldehyds Symptome auftraten, die anch nur im entferntesten an die der Methylalkoholintoxikation erimern. Die physiologische Wirkung des Formaldehyds, die sich in erster Linie als eine sehr stark lokale und allgemeine Reizwirkung darstellt, widerspricht der so naheliegenden Vermutung, daß bei der Oxydation 
des Methylalkohols im Tierkörper beträchtlichere Mengen von Formaldehyd auftreten.

Ueber die Grundsubstanz und die Art der Entstehung ller durch die Nieren mit dem Harne ausgeschiedenen normalen A, $m$ e is $e$ in s ä $u$ r. e sind verschiedene Vermutungen ausgesprochen und von verschiedenen Seiten auch bestimmtere Angaben gemacht worden. Nach Poh l tritt Bildung von Ameisensäure im Tierkörper nux nach Darreichung von. Methylderivaten wie von Methylilkohol auf, nicht aber von solchen Substanzen, welche die Methylgruppe an Kohlenstoff gebunden enthalten, wie dies beim Aethylalkohol und Aceton der Fall ist. Dieser Anschaumng entspreche auch die Tatsache, daß Kohlenhydrate, Fette und Eiweißstoffe unserer Nahrung keine Ameisensïurebildung veranlassen. - Nach Versuchen von $H, v$. Ho e $B\left(\mathrm{in}^{1}\right)$ ist $\mathrm{Ch}$ ol in, das Kaninchen per os oder'subkutan beigebracht wird, im Harn der Tiere niemals als solches wiederzufinden. Die normale Ameisensäuremenge zeigte bei den Versuchstieren eine ziemlich große Inkonstanz; trotzdem war der Einfluß der Cholinzufuhr bei den Kaninchen auf die Ameisenxäureausscheidnng kaum zn verkennen. Da Amcisensäure in go1. ingerer Menge im normalen t"arne vorkommt, reichlicher im Harne von Fiebernden und Lenkïmikern, nimmt $v$. Hoe Blin an, dabs sie gerade in diesen Fällen nicht nur aus dem Cholin der Nahrung, sondern auch aus dem $\mathrm{L}$ e $\mathrm{c}$ i t $\mathrm{h}$ i n zerfallender Zellen herrührt. Steppuhn und $S c h$ ellbach haben bei Funden nach Verfütterung von $\operatorname{Tr}_{\mathrm{r}} \mathrm{n} b \mathrm{en} \% \mathrm{u} \mathrm{cker}$ eine Vermehrung der Ameisensäure im Harn der 'Tiere beobachtet, während sich das gleiche bei Kaninchen nicht nachweisen ließ. Nach dem Frgebnissc: bei dem Hunderersuch halten die genannten Autoren einen inneren Zusammenhang zwisehen der Tranbenzuckerzufuhr und der Ameisensïureausscheidung für erwiesen. A b s o l a t genommen hält sich aber die Ameisensäureausscheidung doch in recht beschei. d e n e $n$ Gr e n z e n, denn an den Versuchstagen stieg bei Trauben. zuckerfütterung bei dem Versuchshunde die normale tägliche Ameisensäureausscheidung von $8,7 \mathrm{mg}$ auf etwa $13,8 \mathrm{mg}$ täglich. E. S a l kow s k i3) nimmt für diese Ameisensiäurebildung aus Traubenzucker als Zwischenstufe den Formald ehyd an, der wuch außerhalb des tierischen Organismus durch Permanganatoxydation aus. Traubenzucker entsteht und zieht ferner auch das O l y c e r in als Vorstufe fül die Ameisensäurebildung im tierischen Organismus in Frage. Glycerin wird bekanntlich regelmäBig im Darmkanal frei und zwar durch Spaltung der Fette. R o s entha le $\mathrm{r}^{4}$ ) und E. Salkow s $\mathrm{i}^{5}$ ) haben gleichzeitig und unabhängig voneinander gefunden, daß Glycerin bei der Oxydation durch Kalinmpermanganat reichlich Formaldehyd liefert. S a I-

$27(1906)$.

1) Beiträge zur chemischen Physiologie und Pathologie Bd. 8,

2) Zeitschr. f. physiol. Chemie 80, 275 (1912).

3) Biochemische Zeitschrit t Bd. 67, 354 (1914).

t) Archiv der Pharmazie Bd. 251. 587 (1914).

5) Zeitschr. f. Tintersuchung d. Nahrungs- und cenuBnitte? B.t. 25. 225 (1914) und Biochemisehe Zejteclu. 67. 354 (1914). 
k ow sk i (l. e.) hat nun bei einer Hündin, die an drei anfeinanderfolgenden Tagen je $10 \mathrm{~g}$ und am vierten Tage $20 \mathrm{~g}$ Glycerin, in den Magen eingeführt, erhalten hatte, eine. der absoluten Menge nach, freilich versel wind end kleinesteiger ung der Ameisensäureausscheidung benbachtet, nämlich eine solche von etwa $7 \mathrm{mg}$ pro Tag. Auf die verfütterte Glycerinmenge bezogen. betrug die vermutlich aus dem Glycerin hervorgegangene Menge

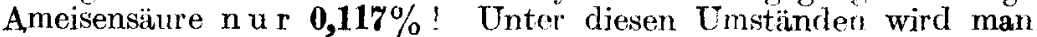
doch noch an andere Quellen für die Bildung der im Harne auftretenden Ameisensäure zu denken haben. -- F r a n $\mathrm{ch} \mathrm{in} \mathrm{j}^{1}$ ) will nach Zufuhr von Is e i t b in eine Ameisensäurebildung im Organismus des Kaninchens beobachtet haben, ohne aber vorher die normale Ameisensäureausscheidung dieser 'Tiere clurch die Niere ermittelt zu haben. E. S a $l k$ o $w i k$ hat diese Normalansscheidung beim Kaninchen, die eine recht sihwankende sein kann, nachträglich bestimmt und ist hierdureh $z u$ der Ansicht gelangt. daß die Zunahme

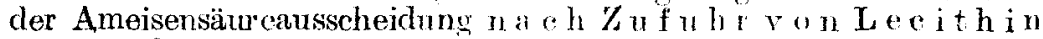
nieht bewiesen sei. - Nalkowski kommt zu dem Schlusse, daß kein Grund zu der Anmahme vorliegt. daß die im Harne der Tiere auftretende Ameisensïture nur ans ein er Substanz hervorgehen soll. Warum sollten nicht verschiedene Quellen für die Ameisensäurebildung - - wie die Kohlenhydrate. dass Glycerin und schlieBlich aneh das Cholin in Frage kornmen?

Sieht man von einigen Bestimmungen der Ameisensiaure in verschiedenen normalen und pathologischen menschlichen Harnen $\mathbf{a b}$, so haben die angeführten Antor en ihre Versuche nur an $\mathrm{T}$ i e r $\in$ :n angestellt, in deren Harn der Amejsensäuregehalt festgestellt wurde. Seit den Massenvergiftungen Berlinel Asylisten dureh Methylalkohol hat das Schicksal dieses Alkohols im me ns eh li e h e n K ̈̈r per sowie die Entstehung etwaiger Oxydationsprodukte in demselben und die Ausscheidung der letzteren mit dem Har'n trhöhtes Interesse? erlangt. Zur Aufklärung dieser Fragen habe jeh im Auschlusse an die in ejner früheren Abhandlung (1.) niedergelegten Fugebnisse die im folgenden beschriebenen Versuche angestellt. Fis sollte durch Untersuching einer größeren Anzahl Proben von verschicdenen menschlichen Harnen zunächst festgestellt werdert. ob Ameisensäure in der Tat als ein $n$ or m i ler Bestandteil don Menchentharns getten kann. ferner. falls dies zutrifft, ob die von einer bestimmten Person bei einigermalien gleichbleibunder Finnährungsweise innerhalb einer bestimmten Zeit ausgeschiedenen Ameisensäuremengen konstant sind, oder ob dieselben größeren Soh wankungen unterliegen und zwar innerhalb weleher Grenzen. Endlich sollte bei einer Person, deren mittlere Ameisensiur eausscheidung trmittelt ist, durch Versuche festgestellt werden, ob die innerliche Darreichung bestimmter Stoffe auf diese Ausscheidnng einen bestimmten Einflu $B$ ausübt und, falls dies zutreffen sollte, in welchem Umfange. Es wurden Methylalkohol. Formaldehyd in Form von Urotropin, a meisensaures und milchsares Natrium sowe Traubenzucker in den Kreis meinet

3) Biochemische \%eitschr. Bd. 6. 270 (1907). 
Untersuchungen gezogen. Diese Untersuchungen konnten unter Umständen auch über die Art der Entstehung der Ameisensäure im menschlichen Körper Aufschluß geben und in erster Linie auch darüber, ob Milchsäure und Traubenzucker als eine V or st u f e für die Ameisensäurebildung daselbst in Betracht kommen können, wie dies von verschiedenen Seiten angenommen wird.

A. J u c ke $n \mathrm{a} \mathrm{ck}^{1}$ ) hat in einem Vortrage über seine Untersuchungen anläßlich der Berliner Massenvergiftungen durch Methylalkohol erwähnt, , daß in dem Harne einer. Leiche, deren Leber und Darminhalt Methylalkohol enthielten, Ameisensäure nachgewiesen werden konnte". Obgleich es Jucken a ck nicht ausgesprochen hat, liegt nach diesen Angaben die Annahme nahe, daß bei einer vermuteten Methylalkoholvergiftung unter Umständen schon der qu a litative Nachweis der Ameisensäure im Harne der vergifteten Person genügen könnte, um mit Bestimmtheit auf eine Vergiftung durch Methylalkohol schließen zu können. Auch zur Aufklärung dieser Frage werden die Ergebnisse meiner Versuche einen kleinen Beitrag liefern.

\section{Zur Methodik der Ameisensäurebestimmung im Harn.}

Alle Autoren, die sich bisher mit derartigen Bestimmungen befaßt haben, geben übereinstimmend an, daß Ameisensäure, obgleich deren Siedepunkt bei $101^{\circ}$ liegt, mit Wasserdämpfen außerordentlich langsam überdestilliere. $H$. F r a $n z$ e $n$ und $G$. $G$ r e v e $e^{2}$ ) wie auch $O$. St e p p u h n und $H$. Seh ellba $\mathrm{Sh}^{3}$ ) führen daher die Destillation im lebhaftesten Wasserdampistrome aus. - Für die quantitative Bestimmung der Ameisensäure dient fast ausschließlich die Methode von Scala-L i ebent), die auf der Reduktion des Quecksilberchlorids zu Kalomel, der gewogen wird, beruht:

$$
\mathrm{CO}_{2} \mathrm{H}_{2}+2(\mathrm{ClHgCl})=\mathrm{CO}_{2}+2 \mathrm{HCl}+\mathrm{Hg}_{2} \mathrm{Cl}_{2} \text {. }
$$

Da im Harndestillate außer Ameisensäure manchmal auch andere, Quecksilberchlorid ebenfalls reduzierende Stoffe wie A 1 t e h yd e, rorhanden sind, kann man dasselbe nicht direkt mit Quecksilberchlorid in Reaktion bringen, sondern es muß erst mit einem kleinen Ueberschusse von Calciumkarbonat eingedampft werden. Wenig Beachtung hat bisher eine Bemerkung von $G$. F r a n e h in $i^{5}$ ) gefunden, daß sich nämlich im Destillate des Harns s tets eine gewisse Quantität sch wefliger Sä u re vorfindet. Der bei Nichtberücksichtigung der schwefligen Säure gemachte Fehler beträgt bis $0.017 \mathrm{~g}$ Ameisensäure pro Tag. Durch die von uns angewandte $\mathrm{Ca} 10 \mathrm{im} \mathrm{marbonatmethode}$ wird die sch weflige Säure ausgefällt oder das Sulfit beim Eindampfen zu Sulfat oxydiert. - Ferner ist daranf zu achten, daß ein be -

1) Zeitschr. f. Untersuchung d. Nahrungs- and Genußmittel

Bd. 24, 7 (1912).

$\left.{ }^{2}\right)$ Zeitschr. f. physiolog. Chemie Bd. 64, 169 (1910).

3) Ebenda, Bd. 80, 274 (1912).

4) Monatshefte für Chemie 14, 747.

5) Biochemische Zeitschrift Bd. 6, 218 (1907). 
d e u t end er Uebenschuß von Quecksilberchlorid angewandt und das betreffende Gemisch mehrere Stunden im Wasserbade erwärmt wird. - Endlich gehen bei der Destillation von Menschenharn öfters Substanzen über, die mit Quecksilberchlorid flockig weiße. $\mathrm{n}$ i $\mathrm{c} \mathrm{h} \mathrm{t}$ durch Ameisensäure bedingte Niederschläge geben, die aber von Salżsäure gelöst werden, während Kalomel bei $\mathrm{n} \mathrm{ich} t$ z u starker Konzentration der Säure praktisch ungelöst bleibt. Franzen and Gr eve (l. c.) schreiben: ,Nachdem sich (ler Niederschlag (von $\mathrm{Hg}_{2} \mathrm{Cl}_{2}$ ) wieder vollkommen zu Boden gesetzt hat, werden $20 \mathrm{cem} \mathrm{k} \mathrm{onzentrier} \mathrm{te} \mathrm{Salzsäure} \mathrm{hinzugefügt.}$ gut umgerührt und nochmals eine Stunde lang in das Wasserbar gestellt." Unter konzentrierter Salzisäure wollen die beiden Verfasser wohl die bei $15^{\circ}$ gesättigte, $42,9 \% \mathrm{HCl}$ en haltende rauchendc Salzsäure des Laboratoriums - die freilich selten stärker als $38 \%$ ig sein dürfte - verstanden wissen. Nun gibt $\mathrm{R}$. F r e s e n i u $\mathbf{s}^{1}$ ) an: ,Sehr vercünnte Salzsäure löst das Quecksilberchlorür bei gewöhnlicher 'Temperatur nicht, bei erhöhter langsam, in der Siedehitze, unter Mitwirkung der Luft, allmählich vollständig; dic Lüsung enthält dann. Quecksilberchlorid: $\mathrm{Hg}_{2} \mathrm{Cl}_{2}+2 \mathrm{ClH}+\mathrm{O}=$ $2 \mathrm{HgCl}_{2}+\mathrm{H}_{2} \mathrm{O}$. Kochende konzentrierte Salzsäure zersetzt das Quecksilberchlor ür ziemlich schnell in zurückbleibendes Quecksilber und sich lösendes Chlorid." Nach diesen Angabers von R. Fresenius sollte man annehmen, daß beim Arbeiten nach der von Fra $\mathrm{n} z$ en $\mathrm{n}$ u $\mathrm{Gr}$ eve gegebenen Vorschrift eine gewisse Menge Quecksilberchlorür in Lösung ginge, daß somit $\mathrm{zu}$ wenig Ameisensäure gefunden werde. Zur Aufklärung dieses Widerspruchs habe ich den folgenden Versuch angestellt. Das aus $\mathrm{lg}$ Quecksilberchlorid mittels Ameisensäure erhaltene Chlorür wurde mit kaltem Wasser chlorfrei gewaschen, dann mit $600 \mathrm{ecm}$ Wasser angerührt, $10 \mathrm{ccm}$ konzentrierte Salzsäure zi1gesetzt, dann das Gemisch eine Stunde lang im Wasserbade erwärmt. Unter ungefähr denselben Konzentrationsverhältnissen lassen Fra nz e n und Gr e v e die Salzsäure auf den Quecksilberchlorürniederschlag einwirken. Nun wurde abfilıriert und das Filtrat mit Schwefelwasserstoff gesäti igt; es entstand ein schwarzer Niederschlag von Quecksilbersulfid, der nach dem Auswaschen mit Wasser, Alkohol und Aether und nach dem Trocknen bei $110^{\circ}$ $0,0423 \mathrm{~g}$ wog; diese Menge HgS entsprich' 0,044 g Quecksilberchlorür. Aus $1 \mathrm{~g} \mathrm{HgCl}$ erhält man theoretisch $0,87 \mathrm{~g} \mathrm{HgCl}$; von diesen sind bei dem angestellten Versuche $0,044 \mathrm{~g}$ oder $5 \%$ desselben in Lösung gegangen. Ein Verlust ron $0,044 \mathrm{~g} \mathrm{HgCl}$ kommt bei den großen Mengen von 2,5 bis $9,6 \mathrm{~g}$ Kalomel, wie sie von Franzen und Greve gewogen wurden, zumal im rinblick aut den kleinen Faktor 0,0977, mit dem ein exhaltene: Kalomelniederschlag multipliziert werden $\mathrm{muB}$, $\mathrm{rm}$ die entsprechende Mens Ameisensäure zu erfahren, kaum in Betracht. Bei den meist erheblich kleineren Kalomelniederschlägen, wie ich sie bei meinen Unter suchungen erhalten habe, hätte ein Substanzverlust von $44 \mathrm{mg} \mathrm{Hg('l}$

1) Anleitung zur quantitativen chemischen Analyse. VI. Auflage (1875). T. Bd., Seite 182 . 
schon einen größesen Fehler verursacht. Ich habe daher die erhaltenen. Kalomelniedeischläge, je nach ihrer: Menge, nur mit 1,2 orler höchstens $3 \mathrm{cem}$ 20\%iger Salzsäure eine halbe Stunde lang im Wasserbade erwärmt. Bei dieser Säurekonzentration kommt die Löslichkeit des Quecksilberchlo ürs in warmer Salrsäure kaum in. Betracht. - Statt die Ameisensäure in einem lebhaften Wasserdampfstrome abzudestillieren, was bei einer $q u$ a $n$ i t a $t$ i v en Bestimmungsmethode eine peinliche Ueberwachung der Destillation erfordert, liabe ich es meist vorgezogen, die Destillation des Harns unter Ersatz des abdestillierten Wassers so lange fortzusetzen, bis alle Ameisensäure übergegangen war. Nur in der letzien Zeit bei lem schlechten Gasdrucke habe ich die Destillation in einem Wasserdampfstrome vorgenommen und hierbei jeweils gegen 21 Destillat in einer Aufschlämmung von Caleinmkarbonat in Wasser aufgesammelt.

A usführung. $\quad 300$ cen Harn werden in einem $3 / 41$

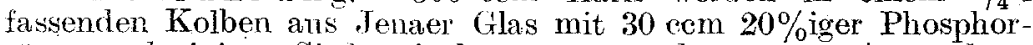
saure und einigen Siedesteinchen versetzt, dann unter Anwendung eines Aufsatzes bei l eb haftest e m S i ed e $\mathbf{n}$ der. Destillation unterworfen. Man verwende einen Tech- oder zwei einfache Bunsenbrenner. Nur za Beginn des Siedens macht sich bei vielen. Harnen ein lästiges Aufschäumen bemerkbar - man erhitze daher die ersten zehn Minuten nur mit kleiner Flamme - das aber nur kurze Zeit anhält, um damn einem durchaus ruhigen Kochen des Harn-Phosphorsäuregemisches Platz zu machen. Das Destillat fängt man in einem Meßzylinder auf: sind $300 \mathrm{ccm}$ übergegangen, so bringt man in den Destillierkolben $300 \mathrm{ccm}$ Wasser, destilliert die gleiche Menge ab und fährt in dieser Weise fort, bis das Destillat nicht mehr sauer reagiert. Dieser Punkt ist fast immer erreicht, wenn $1200-1500 \mathrm{ccm}$ Destillat übergegangen sind. Um sicher zu gehen, kann eine Probe des zuletzt erhaltenen Destillats mit Quecksilberchlorid auf Kalomelbildung untersucht werden; man lasse dieselbe mindestens zehn Minuten lang im siedenden Wasserbade stehen; sie darf hierbei höchsiens ganz sohwach getüubt werden. Das aufgesammelto Desillat wird mit einem Ueberschusse einer Aufschlämmung von Calciumkarbonat in Wasser gut verrührt, auf dem Wasserbade auf etwa $20 \mathrm{com}$ eingedampft, abfiltriert und das Filtrat mit $50-60 \mathrm{ccm}$ einer kalt gesättigten Quecksilberchloridlösung unter häufigem Umschütteln fünf bis sechs Stunden im Wasserbade erhitzt, dann werden, je nach der Größe des Niederschlags, $1-3 \mathrm{ccm}$ Salzsäure von $20 \%$ HCl zugesetzt, umgeschüttelt und nochmals etwa 20 Minuten im Wasserbade erwärmt. Der entstandene Kalomelniederschlag wird auf einem, bej $100-\mathbf{1 0}^{\circ}$ getrockneten und gewogenen Filter oder in einem Gochtiegel gesammelt, mit Wasser chlorfrei gewaschen, mit Alkohol and Aether nachgespült and eine Stunde bei $100-110^{\circ}$ getrocknet. Zur Umrechnung auf Ameisensäure ist das Gewicht des erhaltenen Kalomelniederschlags mit 0,0977 zu multiplizieren.

Zur Ermittelung der Genauigkeit des Verf a h rens diente eine verdünnte wässerige Ameisensäure, deren 
Gehalt direkt mit Quecksilberchlorid, dann nach dem Eindampfen mit viel Wasser und überschüssigem Calciumkarbonat und endlich nach vorausgegangener Destillation ermittelt wurde.

a) $\mathbf{1 0} \mathrm{cem}$ der Säure lieferten, nach vorausgegangener Neutralisation mit Natriumkarbonat, $0,880 \mathrm{~g} \mathrm{HgCl}=0,086 \mathrm{~g} \mathrm{CH}_{2} \mathrm{O}_{2}$.

b) $10 \mathrm{cem}$ derselben Ameisensäure lieferten, mit überschüssigem Calciumkarbonat und $1200 \mathrm{ccm}$ Wasser auf $30 \mathrm{~cm}$ eingredampft, dann filtriert, $0,884 \mathrm{~g} \mathrm{HgCl}=0,0867 \mathrm{~g} \mathrm{CH}_{2} \mathrm{O}_{2}$.

c) $10 \mathrm{ccm}$ derselben Ameisensäure, mit $30 \mathrm{ccm}$ Phosphorsäure und $300 \mathrm{ecm}$ Wasser wiederholt destilliert und $1500 \mathrm{ccm}$ Destillat aufgesammelt, lieferten $0,881 \mathrm{~g} \mathrm{HgCl}=0,0862 \mathrm{~g} \mathrm{CH}_{2} \mathrm{O}_{2}$.

Bestimmung der ,normalen" Ameisensäure im menschlichen Harn.

Zunächst wurde die normalerweise im Harne von sechs verschiedenen Personen vorhanden gewesene Ameisensäure bestimmt, und zwar wurde die Menge an Ameisensäure anfänglich auf die in 24 Stunden gelassene Harnmenge, bei späteren Versuchen auf die Menge Harn von zwei Tagen bezogen. Der innerhalb zweimal 24 Stunden gelassene Harn wurde gut gemischt, gemessen und die Ameisensäure in jeweils $300 \mathrm{ccm}$ desselben in der angegebenen Weise bestimmt. Von der einen Person, die zu weiteren Versuchen herangezogen wurde, ist die normale A,meisensäuremenge wiederholt bestimmt worden, ym einen verwertbaren Durchschnittswert für dieselbe zu erhalten.

I. Harn von einer erwachsenen männlichen Yerson.

a) Harn vom 18. November 1918. Nenge: $1800 \mathrm{ecm}$. Destillat: $1.400 \mathrm{cem}$. Gewogen : $0,7822 \mathrm{~g} \mathrm{FrCl}=0,0766 \mathrm{~g} \mathrm{CH}_{2} \mathrm{O}_{2}$.

Ausscheidung in der Tagesmenge: $0,344 \mathrm{~g}$ Ameisensäure.

b) Harn vom 19. November 1918. Menge: $1280 \mathrm{cem}$. Destillat: 1500 cem. Gewogen: $0,586 \mathrm{~g} \mathrm{HgCl}=0,0573 \mathrm{~g} \mathrm{CH} \mathrm{CH}_{2}$.

Ausscheidung in der Tagesmenge: 0,2291 g Ameisensäure.

In $\mathrm{z}$ we i Tagen sind mit dem Harn $0,344+0,229 \mathrm{l}=0,574 \mathrm{~g}$ Ameisensäure ausgeschieden worden.

c) Harn vom 20. und 21. November 1918. Yenge: 3300 crm. Gewogen: $0.604 \mathrm{~g} \mathrm{HgCl}=0,0591 \mathrm{~g} \mathrm{CH}_{2} \mathrm{O}_{2}$.

Ausscheidung von zwei Tagen: $0,6491 \mathrm{~g}$ Ameisensäure.

d) Harn vom 17. und 18. Februar 1919. Menge: $4800 \mathrm{cem}$. Gewogen: $0,382 \mathrm{~g} \mathrm{HgCl}=0,03733 \mathrm{~g} \mathrm{CH}_{2} \mathrm{O}_{2}$.

Ausscheidung von zwei Tagen: $0,597 \mathrm{I}^{2} \mathrm{~g}$ Ameisensäure. Kontrollbestimmung für die gleiche Zeit: $0, \tilde{5} 72 \mathrm{~g}$ Ameisensäure.

e) Harn vom 24. und 25. Februar 1919. Menge: $3500 \mathrm{~cm}$. Cewogen: $0,440 \mathrm{~g} \mathrm{HgCl}=0,04,299 \mathrm{~g} \mathrm{CH}_{2} \mathrm{O}_{2}$.

Ausscheidung von zwei Tagen: $0.5034, \mathrm{~g}$ Ameisensäure.

f) Harn vom 4. und 5. März 1919. Menge: $4500 \mathrm{ccm}$. Gewogen: $0.376 \mathrm{~g} \mathrm{Hg}=0,036736 \mathrm{~g} \mathrm{CH}_{2} \mathrm{O}_{2}$.

Ausseheidung von zwei Tagen: $0,551 \mathrm{~g}$ Ameisensäure.

g) Harn vom 10. und 11. März 1919. Menge: $3300 \mathrm{ccm}$. Gewogen: $0,465 \mathrm{~g} \mathrm{HgCl}=0,04543 \mathrm{~g} \mathrm{CH}_{2} \mathrm{O}_{2}$.

Ausscheidung von zwei Tagen: 0,499 (abgerundet 0,5 g) Ameisensäure. Kontrollbestimmung: $0,463 \mathrm{~g}$ Ameisensäure. 
Der besseren Uebersichtlichkeit halber lasse ich die jeweils tor zwei Tage gefundenen n or mal en Ameisensäurewerte, tabellarisch zusammengestellt, nochmals folgen:

\section{Zeit: Diurese:}

19. und 20 . September 1918

21. und 22. September 191.8

17. und 18. Februar: 1919

24. und 25. Februar 1919

4. und 5. März 1919

10. und 11. Värz 1919
$3080 \mathrm{cem}$

$3300 \mathrm{cem}$

$4800 \mathrm{ccm}$

$3500 \mathrm{ccm}$

$4500 \mathrm{ccm}$

$3300 \mathrm{ecm}$
Ameisensäuregehalt rer 48 stündigen Harnmenge

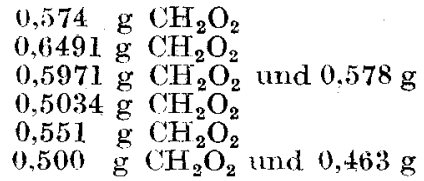

Aus dieser Zusammenstellung ist zu ersehen, daß die Aus*heidungsgröße der Ameisensäure bei der betreffenden Person innerhalb von vier Monaten keinen allzugroßen Sehwankungen unterworfen war. Hängt die Bildung der Ameisensäure im menschlichen Organismus und demnach auch ihre Ausscheidung mit der Art der Frnährung zusammen, so ist es bei der zur Zeit ziemlich gleichbleibenden, wenig Abwechselung bietenden, eiweiß- und fettarmen, andererseits kohlenhydratreichen Kost nicht zu verwundern, wenn die Ausscheidungsyröße der Ameisensäure bei ein und derselben Person für längere Zeit ziemlich konstant bleibt. Man kann schon sagen, daß sich die in Frage kommende Versuchsperson im, A me is e n s äu r e gleichgewicht" befunden hat, und daß sich dieselbe aus diesem Grunde für die von mir beabsichtigten Versuche besonders gut eignete. Sie hat innerhalb von zwei Tagen, als Mittel von sechs, zu verschiedenen Zeiten ausgeführten Bestimmungen, $0,562 \mathrm{~g}$ oder in 24 Stunden $0,281 \mathrm{~g}$ Ameisensäure mit dem Harne ausgeschieden.

it. Harn von einem geschlechtskranken Manne; diesen Harn verdanke ich der gütigen Vermittelung des Herrn Hiofrat Dr. med. $\mathrm{T}$ a e ge. Harnmenge von zwej Tagen: $4600 \mathrm{~cm}$. Gewogen: 0,4721 $\mathrm{g}$ $\mathrm{HgCl}=0,0462 \mathrm{~g} \mathrm{CH}{ }_{2} \mathrm{O}_{2}$.

Ausscheidung von zwei Tagen: $0.7101 \mathrm{~g}$ Ameisensäure, Kontrollbestimmung: $0,7065 \mathrm{~g}$ Ameisensäure.

III. Harn von einem an Arteriusklerose erkrankten Manne der hiesigen medizinisehen Klinik.

a) Farn vom 10. und 11. März 1919. Menge: $4700 \mathrm{cem}$. Gewogen: $0,082 \mathrm{~g} \mathrm{HgCl}=0,008 \mathrm{l} \mathrm{g} \mathrm{CH}_{2} \mathrm{O}_{2}$.

Ausscheidung von zwei Tagen: $0,127 \mathrm{~g}$ Ameisensäure.

b) Harn vom 20. und 21. Wärz 1919. Menge: $4400 \mathrm{~cm}$. Gewogen: $0,078 \mathrm{~g} \mathrm{HgCl}=0,0077 \mathrm{~g} \mathrm{CH}_{2} \mathrm{O}_{2}$.

Ausscheidung von zwei Tagen: $0,114 \mathrm{~g}$ Ameisensäure.

IV. Haru von eimem 28jährigen gesunden Studenten.

Menge von zwei Tagen: $3800 \mathrm{~cm}$. Gewogen: $0,146 \mathrm{~g} \mathrm{HgCl}=$ $0,0143 \mathrm{~g} \mathrm{CH}_{2} \mathrm{O}_{2}$. Snmit Ausscheidung in zwei Tagen: $0.182 \cdot \mathrm{g}$ Ameisensäure.

Außerdem wurde die Ameisensäure in einer größeren Anzahl Harnproben' verschiedener Herkunft qua lita $\mathrm{t}$ iv nachgewiesen; hierbei habe ich keinen einzigen Harn in die Hände bekommen, der wenigstens nicht Spuren von Ameisensäure enthalten hätte 
Nach diesen Bofunden scheint mir die $S$ ch $I$ u $\beta$ f olger ung. raß Ameisensäure ein normaler Bestandteíl des mensehlichen Harns ist, durohans be. rechtigt zu sein. Da die Probe mit Quecksilberchlorid fïr Ameisensïure nicht spez if isch ist, obgleich ja bei der angegebenen Arbeitsweise verschiedene anderc, ebenfalls reduzierend wirkende Stoffe wie Aldehyde und schweflige Süure ausgeschlossen sind, habc ich es doch für angebracht gehalten, die Anwesenheit der Aneisensiture durch Teberführung derselben nach F e $n$ t on und $\mathbf{8}$ i s s o $\mathbf{n}$ in saurer Lijsung mittels Maynesiumspänen in Form. aldehyd und Nachweis dieses, besonders festzustellen. E's ist mi in dor Tat verschiedene Male gelungen. in verschierlenen Harnproben der im Ameisensïuregleichgewicht befindlichen Person, nach vorausgegangener Reduktion nach $F$ e n t on-S is s on. mit Hilfe von Morphin-Schwefelsïure, von Fuchsinschwefligsiinte, nach der Hehner'schen Fchichtjorobe wic auch nach der Prohe des Fleisch. beschaugese zes von 30. Jamuar 1903 Formaldehyd bestimmt nachznweisen. Nach der letzteren Probe soll die fragliche Flüssigkeit mit frischer ungekochter Milch und Eisenchlor ifl haltender Salzsäure wïhrend einer halben Minute gelinde zum Sieden erhitzt werden. Leh habe gefunden, daß diese Probe bei $1 \ddot{a} n \mathrm{ng}$ er anhaltendem, s:hwachen Sieden, während ein bjs zwei Minuten, bedeutend empfindlicher wird. Zum sicheren Nachweis der Ameisensiaure durch Reduktion zu Form. aldehycl, die keineswegs quantitativ verlauft, habc ich jc $300-\tilde{n} 00 \mathrm{ccm}$ Harn abdestilliert und das ganze aufgesammelte Destillat mit überschüssigen Calciumkarbonat auf $10 \mathrm{ccm}$ ein. gedampft, dann abfiltriert, das Filtrat mit $5 \mathrm{ccm}$ Salzaïure (spez. Gewicht 1,124 ) versetzt und allmählich $1-2$ g Magnesiumspäne. eingetragen.

Dip Bestimmung des Ameisensäuregehaltes dex Harns naeh Winnahme von Methylalkohol.

1)ie Versuchsperson mit der ziemblich konstanten Ausscheidun: von $0,562 \mathrm{~g}$ Amejsensiiure für zwei Tage nahm in: Verlaufe Fol zwei Tagen $25 \mathrm{~g}$ reinen Methylalkohol ein und zun mit himh(oy. sirup-haltigem Wasser auf $1 / 21$ verdünth.

a) Harn von de in beiden ersten Versuchs. t ag en, nämlich vom 9 . und 10. Januar 1919, an clenen die 25 \& $\mathrm{Cr}_{4} \mathrm{O}$ eingennmmen wurdell. Harnmenge: $3300 \mathrm{ccm}$. Verarbeitet: wjo immer $300 \mathrm{ccm}$ Harn. Gewogen: $0,488 \mathrm{~g} \mathrm{HgCl}=$ $1,0477 \mathrm{~g} \mathrm{Cir}_{2} \mathrm{O}_{2}$. Somit Ausscheirung von zwoi Tagen: $0.525 \mathrm{~g}$ Ameisensiture.

Weiterc Proben desselben Hains wurden auf einen Gehalt un Methylalkohol und Formaldehyd geprift, die. uber nicht nachgewiesen werden konnten.

Zur Prüfung auf Methylalkoholgehalt diente die von A. Jucken a ck (1. c.) angegebene Probe; um die Empfindlich. keit derselben kennen in lernen, wurden $10 \mathrm{ccm}$ cles Holzgeist und Formaldelyddrei befundenen Harndestillates mit einem Tröpfchen Me hylalkohol versetz.t unil alsdann oxyiliert: jetzt fielen dic Proben 
mit Fuchsinschwefligsäure. mit Morphin-Schwefelsäure und mit Milch + eisenchlorjdhultige - Salzsäure stark positiv aus.

b) Harn von den beiden folgenden Tagon, Ii $\mathrm{m} \mathrm{lich}$ v o m 11. und 12. Januar 1919, an denon cbenfalls zusammen $25 \mathrm{~g}$ Methvlalkohol eingenommen wurden. Harumenge: $3600 \mathrm{ccm}$. Angewandt: $300 \mathrm{ccm}$ Harn. (iewogen: $1,662 \mathrm{~g} \mathrm{HgCl}=$ $0,1644 \mathrm{H} \mathrm{CH}_{3} \mathrm{O}_{2}$. Somit Ausschedung von zwei Tagen: 1,978 $\mathrm{g}$ Ameisensäure. Me hylalkohol und Formaldehyd ließen sich anch in diesem itarndestillate nicht nachweisen.

c) Harn vonden beiden folgenden Tagen, uämlich rom 13. und 14. Janurr 1919. An diesen beid'n Tagen wurde kein Mcthyla lkohol eingenommen. Harnnenge: $4500 \mathrm{ccm}$. Angewandt: $300 \mathrm{ccm}$ Harm. Gewogen: $0,9332 \mathrm{~g} \mathrm{HgCl}=$ $0.09117 \mathrm{~g} \mathrm{CH}_{3} \mathrm{O}_{3}$. Ausssheirlung von zwei Tngen: $1,37 \mathrm{~g}$ Amejensilure.

Nach Hinnahme von $50 \mathrm{~g}$ Methylalkohol in Verlaufe von vier Tagen konnten unverändert gebliebener Methylalkohol sowie wein erstes Oxydationsprodukt, der Formaluehyd, in Harn der Verauchsperson $n$ i c h t a.lfgefunden werden. - Bei einem weitcren Versuche wiucle an den beiclen folgenten Tagen, 15. und 16. Januar 1919, die Menge des eingenommenen Methylalkohols anf $30 \mathrm{~g}$ erhöht. Diesmal mußto aus rein iiußeren Grïnclen der Hurn von drei anfeinanderfolgenilen Tagen gesainnielt werden. Harnmenge: $5500 \mathrm{ccm}$. Angewnit: $300 \mathrm{ccm}$ Ha'n. (Gewogen: $2,194 \mathrm{~g} \mathrm{HgCl}^{\circ}=$ $\left.0.2143 \mathrm{~g} \mathrm{CH}_{2} \mathrm{O}\right)$. Somit Ansscheidung von clrci Tagen: 3,946 g Anteisehä̈ure. Ber Harn von diesen Yersuchstagen hat zwny ebenfalls keinen Formaldchyrd, wohl aber e ine Sp u $\mathrm{M}$ e thy la $1 \mathrm{koh}$ ol enthaiten, denn das Harndestillat yah, nach der Permanganaioxydation, die $\mathrm{H}$ ol $\mathrm{n}$ er' 'sche nnd die Iruchsinsel $w$ wefligsäureprobe. wenn auch unr selur schwacl. Mit Fuchsinsrhwefligsizure färbitc sich las oxpdierte Destillat nnch einiger '/ejt deutlich blauviolett. wïhrend drs urspriingliche Destillat mit dem gloichen Rengens. sturlı bei lingerem Stehen, vollkommen ingefärht blieh.

Dic Versuchsperson schied innerhalb von zwej Tagen n or ma ler we i ge 0,5R2 g Ameisensiure aus; diese Menge muß selbstvorstainllich in Abzug gehrncht werden, nm die Vermelurung der Ameisen:xäme nach Fimnahme von Methylalkohol zu orfahren.

Gesarnt-

Zeit: Einnalme: . usswrheidung

1. und 2. Tag

3. und 4. Tag

5. und 6. Tag

7. und 8. Tag
$2 \pi \mathrm{g} \mathrm{CH}_{2} \mathrm{OH}$

$25 . \mathrm{C} \mathrm{CH}_{3} \mathrm{OH}$

$30 \mathrm{~g} \mathrm{CH}_{3} \mathrm{OH}$ $0,525 \mathrm{~g}$

$1,975 \mathrm{~g}$

$1.376 \mathrm{~g}$

$3.945 \mathrm{~g}$
Amcisensälur.
Vormelirung dor susg.

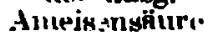

$$
\begin{array}{cc}
1.313 \mathrm{~g} & \begin{array}{c}
\text { zu1- } \\
\mathbf{0 , 7 1 4} \mathrm{g} \\
\mathbf{3 . 1 6 4} \mathrm{g}
\end{array} \\
\begin{array}{c}
\text { sanmen } \\
5,2 \mathrm{~g}
\end{array}
\end{array}
$$

In Verlnufe ron neht Tagen wurden $80 \mathrm{~g}$ roincr Methyl. alkohol in stıurk verdünnter wässeriger Lösung eingenommen uind wälırend diesel Zeit 5,2 g $A$ me isens äure mehr a us üschieden als unter normalen Verhnltaiesen! These Menge Ameisenseiture entspricht abgerundet $5 \% \mathrm{M}$ e th $\mathrm{y} \mathrm{l}$ : a $1 \mathrm{k}$ o h o l, die von den eingennmmenen $80 \mathrm{~g}$ in Körper der hrtreffenden Versuchisperann mi Amciaenväure nxycliert mol als solche 
ausgeschieden wurden. Diese Zahl ist zweifelsohne $\mathrm{z}$ u $\mathbf{u}$ i e d r ig angenommen, da wahrseheinlich auch an den folgenden Tagen nach der letzten Methylalkoholgabe eine erhöhte Ameisensäureausfulur stattgefunden hat. Leider konnte damals der Harn nicht gesammelt werden. -- Meine Versuchsergebnisse lassen aber deutlich erkennen, daß die aw eingenommenem Methylalkohol im mensehlichen Körper gebildete Ameisensiute nur langsan ausgeschieden wird. Obgleich an den beiden ersten Versuchstagen $25 \mathrm{~g}$ Methylalkohol eingenommen wurden, war der Ameisensäiuregehalt des Harns an diesen beiden Tagen nicht vermeht, die Vermehrung machte sich erst am dritten und vierten Tage bemerkbar. Poh 1 (1. c.) hat bei seinen Versuchen mit Hunden die gleiche Beobachtung gemacht. Diese verlangsamte Ausscheidung der Ameisensäure ist entweder auf eine längere Zurückhaltung der bereits gebildeten Ameisensïure zurückzuführen oder aber, was wahrscheinlicher: isi, auf ein längeres Verweilen des Methylalkoliols oder seiner Umwandlungspiodukte im menschlichen. Körper, die nur allmählich an Ameisensäurc oxydiert werden.

Bemerkenswert ist die Tatsache, daB in keiner der Harnproben aus der Versuchsperiode Formald e hy anfgefunden wurde.

Die Bestimmung des Ameisensäuregehaltes des Harns nach Einnahme von amejsensaurem Natrium.

Die Versuchsperson mit der ziemlich konstanten Ameisensiureausscheidung von $0,562 \mathrm{~g}$ für zwei Tage nahm, auf zwei Tage verteilt, $20 \mathrm{~g}$ reines ameisensaures Natrium in wässeriger Lösung ein; diese Menge entspricht 13,5 g Ameisensäure.

a) Harn von den beiden Versurhstagen (10. und 11. April 1919). Harnmenge: $3800 \mathrm{ecm}$. Abdestilliert: $300 \mathrm{ccm}$ Harn. Gewogen: $1,342 \mathrm{~g} \mathrm{HgCl}=0.1312 \mathrm{~g} \mathrm{CH}_{2} \mathrm{O}_{2}$. Somit Ausscheidung an den beiden Versuchstagen: $1,71 \mathrm{~g}$ Ameisensäure.

b) Harn von den beiden $\mathrm{Nach}$ tagen (12. und 13. April 1919), an denen kein ameisensaures Natrium eingenommen wurde. Harnmenge: $4100 \mathrm{ccm}$. Abdestilliert: $300 \mathrm{ccm}$ Harn. Gewogen: $1,36 \mathrm{~g} \mathrm{HgCl}=0,1329 \mathrm{~g} \mathrm{CH}_{2} \mathrm{O}_{2}$. Somit Ausscheidung an den beiden Nachtagen: 1,82 $\mathrm{g}$ Ameisensếure.

An den vier Tagen sind $1,71+1,82=3,53 \mathrm{~g}$ Ameisensäure mit dem Harn ansgeschieden wrden. Die normale Ausfuhr für vier Tage beträgt aber $1,124 \mathrm{~g}$. Ameisensäure; demnach sind nach Einnahme von $13,5 \mathrm{~g}$ Ameisensäure in Form ihres Natriumsalzes $3,53-1,124=2,406 \mathrm{~g}$ Ameisensäure mehr zur Ausscheidung gelangt als unter normalen Verhältnissen. Das sind genau $15 \%$ der engenommenen Ameisensäure, die sich der Oxydation zu Kohlensäure und Wasser entzogen haben. - Den gleichen Wert fand P o h 1 für einen $7,7 \mathrm{~kg}$ sehweren Hund, der nach Einführung ron $1,253 \mathrm{~g}$ Natriumformiat mittels Schlundsonde in den Magen des Tieres in drei Tagen $18 \%$ des eingeführten Formiates ausschied, and $C$. S ch otten hat bei seinen Versuchen mit Hunden nach Verfütterung von Natriumformat sogar bis $26 \%$ der eingegebenen Ameisensïuremenge im Harn der 'Tjere wiedergefanden. 


\section{Bedimmnng des Ameisensäuregehaltes des Harns nach Einnahme von Hexamethylentetramin.}

Von verschjedenen Seiten wird dje Ansicht vertreten, daB die Oxydation des Methylalkohols in tierischen Organismus zu Ameisensäure sich über den Formaldehyd als Zwischenstufe vollziehe. Ist diese Annahme richiig, so miißte Formaldehyd selbst oder eine jede, diesen Aldehyd abspaltènde Substanz wie das Hexamethylentetramin eine vermehrte Ameisensäureausfuhr verursachen. - Úm den Versuch zu einem möglichst eindeutigen zu gestalten, wurde von der betreffenden Person die normale Ameisensäuremenge des Harns sowohl zwei Tage vor als auch zwei Tage nach der letzten Einnahme von Hexamethylentetramin bestimmt.

a) Bestimmung der normalen Amoisengäur ea us s cheidung. Harn yom 2. und 3. Mai 1919. Menge: $3500 \mathrm{ccm}$. Angewandt: $300 \mathrm{cem}$. Gewogen: $0,315 \mathrm{~g} \mathrm{HgCl}=0,0308 \mathrm{~g} \mathrm{CH}_{2} \mathrm{O}_{2}$. Ausscheidung von zwei Tagen: $0,363 \mathrm{~g}$ Ameisensäure.

b) Versu o h stage. An vier Tagen, nämlich am 4., 5., 6. und 7 . Mai 1919 wurden täglich $2,5 \mathrm{~g}$, zusammen also $10 \mathrm{~g}$ Urotropin eingenommen.

-a) Harn von den beiden orsten Versuchs. t a ge $\mathrm{n}$. Menge: $3500 \mathrm{ccn}$. Angewandt: $300 \mathrm{ccm}$. Gewogen: 0,222 g $\mathrm{HgCl}=0,0286 \mathrm{~g} \mathrm{CH}_{2} \mathrm{O}_{2}$. Somit Ausscheidung von zwei Tagen: $0,342 \mathrm{~g}$ Ameisengëure.

Das Destillat aus dem nit Phosphorsäure stark angesäuerten Harne enthielt $r$ e ich li ch For mald oh yd, wie die Proben mit Fuchsinschwefligsäure. mit Morphin-Schwefelsäure und mit Milch in Verbindung mit Ferrichlorid haltender Salzsäure ergeben haben.

ß) Harn rom dritten und vierten Versuchst a ge. Menge: $3300 \mathrm{ccm}$. Angewandt: $300 \mathrm{ccm}$. Gewogen : $0,1290 \mathrm{~g}$ $\mathrm{HgCl}_{\mathrm{g}}=0,0127 \mathrm{~g} \mathrm{CH}_{2} \mathrm{O}_{2}$. Somit Allsscheidung von zwei Tagen: 0,140 $\mathrm{g}$ Ameisensäure.

Auch das Destillat von diesem Harne enthielt reichlich Formaldehyd.

r) Hern ron z wei $\mathrm{Nachtagen}$. (vom 8. und 9. Mai 1019). Menge: $4000 \mathrm{ccm}$. Gewogen: $0,272 \mathrm{~g} \mathrm{HgCl}=0,0266 \mathrm{~g} \mathrm{CH}_{2} \mathrm{O}_{2}$. Ausschoidung von zwei Tagen: $0,366 \mathrm{~g}$ Ameisensäure.

Auch in dem Harn der zwei Nachtage konnte Formaldehyd nachgetwiesen werden.

Diese Versuche haben in eindeutiger Weise ergeben, dab per os zugeführtes $\mathrm{Hexamethylentetramin,die}$ Ameisensänreausscheidung beim Menschen nicht vermehrt; in dem einen Falle wirde sogar eine Vermind er ung der normalen Ameisensäuremenge poobachtet. Hexamethylentetramin dürfte sonit nicht zu den Ameisensäurebildnern im mensohlichen Körper gehören. Nach diesem Versuchsergebrisse ist es auch weing wahrscheinlich, daß die Oxydation des Methylalkohols zu Ameisensäure über den Formaldehyd als Zwischenstufe erfolgt, da dieser schon durch die Magensäure aus dem Urotropin höchstwahrscheinlich abgespalten 
wird (E. Sa $1 \mathrm{kow}: \mathrm{k}^{\mathbf{1}}$ ). Ein Teil des Hexamethylentetramins geht allem Anscheine nach als solches in den Harn über.

Die $10 \mathrm{~g}$ Hexamethylentetramin, die in Verlaufe von vier Tagen per os eingenommen wurden, hätten bei voll s t ä n d ig er Hydrolyse $13 \mathrm{~g}$ Formalchyd greliefert:

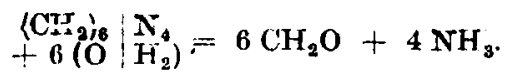

Bestimmung des Ameisensäuregehaltes des Harms nach Einnahute ron milehsaurem Natrium.

Da die gewöhnliche Milchsäure durch hydrolytische Spaltun: in Acctaldehỵd und Ameisensäure zerfällt:

$$
\mathrm{Cil}_{3} . \mathrm{CH}(\mathrm{OH}) . \mathrm{COOH}=\mathrm{CH}_{3} . \mathrm{CHO}+\mathrm{HCOOH} \text {, }
$$

liegt die Annahme nahe, daß Amejsensäure auch im tierischen Organismus aus der in $\mathrm{hhm}$ weit verbreiteten Milchsäure hervorgehe. Steppuhn und. Schellhach (l. c.) schließen sich

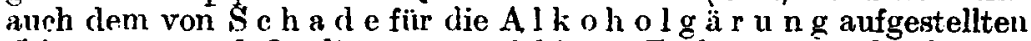
Schema an, daß die gärungsfähigen Zuckerarten durch unbekannte Zwischenstufen auch Milchsiuure bilken, die dann in Sinne der obigen Gleichung in Ase'aldehyd und Ameisensäure zerfällt. Die im annähernden Ameisensäuregleichgewicht befindliche Versuchsperson schien mir geeignet zu sein, die Frage zu entscheiden, ob die Milchsäure im menschlichen Organismus in diese beiden Bestandteile zerlegt wird, und ob dieselbe somit als eine $\mathrm{V}$ or$s \uparrow u f e$ der Ameisensäurebildung angesehen werden darf.

I. a) An zwei Tagen, nämlich am 7. und 8. März 1919, wurden im ganzen $40 \mathrm{~g}$ milchsaures Natrium ejngenommen. Hammenge: $4700 \mathrm{~cm}$. Verwandt: $300 \mathrm{ccm}$ Harn. Gewogen: $0,428 \mathrm{~g} \mathrm{HgCl}=$ $0.0418 \mathrm{~g} \mathrm{CH}_{2} \mathrm{O}_{2}$. Somit Ausscheidung an zwei Perguchstagen: $0,653 \mathrm{~g}$ Ameisensäure.

b) Zwei Nachtage; tiam vom 9. und 10 März 1819. Menge: $4300 \mathrm{ccm}$. Destillat: $300 \mathrm{ccm}$ Ham. Gewogen : $0,589 \mathrm{~g} \mathrm{HgCl}=$ $0,0576 \mathrm{~g} \mathrm{CH}_{2} \mathrm{O}_{2}$. Ausscheidung an den beiden Nachtagen: $0,829 \mathrm{~g}$ Ameisensüure.

c) Zwei weitere Nachtage; Han vom 11. und 12. März 1919. Menge: $3300 \mathrm{ccm}$. Gewogen: $0,469 \mathrm{~g} \mathrm{FgCl}=0,04583 \mathrm{~g}$ $\mathrm{CH}_{2} \mathrm{O}_{2}$. Ausschoidung dieser beiden Nachtage: $0,504 \mathrm{~g}$ Ameisensäure.

II. An zwei Tagen, 3. und 4. April 1919, wurden zusammen 50) $g$ milchsaures Natrium eingenommen.

a) Harn von diesen beiden Versuclistagen. Menge: 4000 ccm. Gewogen : $0,4545 \mathrm{~g} \mathrm{HgCl}=0,0445 \mathrm{~g} \mathrm{CH}_{2} \mathrm{O}_{2}$. Ausscheidung von zwei Tagen: 0,595 g Ameisensäure.

b) Harn von z wei $\mathrm{H}_{\text {ac }} \mathrm{h}_{\mathrm{t}} \mathrm{agen}$. vom 5. und 6. April 1918. Menge: $4800 \mathrm{ccm}$. Gewogen: $0.50 \mathrm{~g} \mathrm{HgCl}=0,04885 \mathrm{~g} \mathrm{CH}_{2} \mathrm{O}_{2}$. Ausscheidung an den beiden Nahlt agen: 0,781 g Ameisensäure.

III. a) Es wurdè zunächst von der Versuchsperson die n o r m a le Ameisensäureausscheidung von zwei Tagen (vom 18. und 19. Februar, bestimmt. Harnmenge: $4800 \mathrm{ccm}$. Gewogen: $0.382 \mathrm{~g} \mathrm{HigCl}=0,0373 \mathrm{~g}$ $\mathrm{CH}_{8} \mathrm{O}_{2}$. Somit Ausseheidung von zwei Tagen: $0,597 \mathrm{~g}$ Aneisensäure eine Kontrollbestimmung ergab $0,571 \mathrm{~g}$ Ameisensëure.

" Biochemische Zeitsclurift 87, 161 (1918). 
b) An zwei Tagen, am 20. und 21. Februar, wurden in ganzen $30 \mathrm{~g}$ milchsaures Natrium eingenommen. Harnmenge: $2900 \mathrm{ccm}$. Gewogen: $1,225 \mathrm{~g} \mathrm{HgCl}=0.1197 \mathrm{~g} \mathrm{CH}_{2} \mathrm{O}_{2}$. Ausscheidung von zwei Tagen: $1.17 \mathrm{~g}$ Ameisensäure.

b) Harn vom ersten und zweiten Nachtage; Hiarn vom 21, und 22. Februar 1919. Menge: $3100 \mathrm{ecm}$. Gewogen: $0.838 \mathrm{~g} \mathrm{HigCl}=0,0819 \mathrm{~g} \mathrm{CH}_{2} \mathrm{O}_{2}$. Ansscheidungen von zwei Tagen: $0.8 \tilde{g} \mathrm{~g}$ Ameisensäure.

c) hiarn vom dritten und vierten Ka tahtage; $\mathrm{vem} \mathrm{23.} \mathrm{und} \mathrm{24.} \mathrm{Februar.} \mathrm{Menge:} 3500 \mathrm{ccm}$. Gewogen: $0.44 \mathrm{~g} \mathrm{HgCl}=$ $0.043 \mathrm{~g} \mathrm{CH} \mathrm{CH}_{2} \mathrm{O}_{2}$. Ausscheidung von zwei Tagen: 0,503 $\mathrm{g}$ Ameisensäure.

Die Ergebnisse dieser Versuche lassen die aufgeworfene Frage nach der Entstehung der Ameisensätre aus Milchsäure im menschlichen Körper nicht eindeutig beantworten. In dem einen Falle stieg ja die normale Ameisensäureausscheidung nach Einnahme von $30 \mathrm{~g}$ milchsaurem Natrium von $0,597 \mathrm{~g}$ auf fast die dop p e l t e Menge, nämlich auf $1,17 \mathrm{~g}$. Andererseits war aber die Steigerung der Ameisensäureausfuht nach Einnahme von 40 und sogar von $50 \mathrm{~g}$ milchsaurem Natrium innerhalb von zwei Tagen außerordentlich gering. In dieser Hinsicht sind noch weitere Versuche nötig, um die Beziehungen zwischen Milchsäurezufuhr per os und Ameisensiurebildung im menschlichen $\mathrm{Or}_{\diamond}$ anismus einwandfrei aufzuklären. Soviel aher läßt sich schon jetz: aus den Frgebnissen meiner Verstche angeben, daß die Gärungsmilchsäure, falls sie als eine Vorstufe für die Ameisensäurebildung $1 \mathrm{~m}$ menschlichen Körper überhaupt in Frage kommt, daselbst nur eine recht g er ing e $\mathrm{M}$ e $\mathbf{n g}$ e Ameisensäure $\pi$ bilden imstande sein wird.

\section{Die Bestimmung des Ameisensäuregehaltes des IIarns nach Finnahme von Trantienzucker.}

Wie bereits erwähnt wurde, haben $\mathrm{Steppuhn}$ und schellbach nach Verfïterung von Traubenzucker bei tunden, nicht aber bei Kaninchen, eine, wenn auch s e h r gering e St e igerung der normalen Ameisensäureausfuhr beobachtet; E. Salkows ki nimmt für diese Ameisensänrebildurw aus Traubenzucker den Formaldehyd als Zwischenstufe an. Es lag für mich der Cerlanke nahe, diesen Versuch mit dem Traubenzucker auf den Menschen zu ïher ragen. Eine erwachsene Vorsuchsperson mit einer normalen Ausscheidung von 0,2 bis $0,29 \mathrm{~g}$ Ameisensäur e für zwei Tage erhielt in Verlaufe von drei Tagen $300 \&$ reinen Traubenzucker. Die Frnährungsweise war während dieser Versuchszeit annähernd die gleiche wie an den versuchsfreien Tagen, nur mit dem Unterschiede, daß Kaffee und Tee nicht mit Süßstoff, sondern mit Traubenzucker versüßt wurden. Da die versüßende Kraft des letzteren erheblich geringer ist wie die des Rohrzuckers, hann schon eine größere Menge Traubenzurcker im Tee celöst werden, bis der süße Teschmack stärker herroririt. Trotz der großen Menge Traubenzucker, die innerhalb ron zwei Tagen dem Magen zageführt wurde, hat sich in Harn Ger Versichsperson keine Spur Zucker nachweisen lassen. 
Harmmenge von den beiden Versuchstagen: 6300 com. Abdestilliert: 300 ccm Harn. Gewogen: $0,074 \mathrm{~g} \mathrm{HgCl}=$ $0,00723 \mathrm{~g}\left(\mathrm{H}_{2} \mathrm{O}_{2}\right.$. Somit Ausscheidung an den beiden Versuchstagen: $0,152 \mathrm{~g}$ Áneisensäure. -- Die Ameisensäureฉusfuhr ist also durch die reichlinhe Wimnahme vom Trambenzucker nicht ge st e i g e $r$ t. sondern im Gegenteil, im Verhältnisse zur normalen Ausscheidumg, ntwas verringert worden. Dieser Abnahme lege ich keme Bedentung bei, da sie noch innerhalb der Sohwankungen, die bei der normalen Ameisensituremsscheirlun! jmmer rorkonimen kënnen, legt.

Im Hinblick auf eine Veröffentlichuny vom $H$. D. D a k in aus neuerer Zeit (vgl. Zustmmenfassung) schien mir ein weiterer Versuch mit Traubenzucker angetzeigt zil sein. Eine-erwachsene männliche Ferson erlielt iuI Verlaufe von 24 stunclen auBer der üblichen Nahrung 150 \& reinen 'Trnvbenzoncker und verspeiste $2 n$ dem reichlich Kartoffeln; wuf jerten Fall cine größere Menge derselben als am Vorversinchistage.

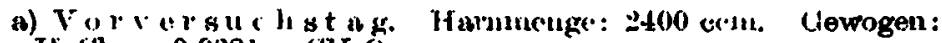
$0,288 \mathrm{~g} \mathrm{FgCl}=: 0,0281$ g $\mathrm{CHF}_{2} \mathrm{O}_{2}$.

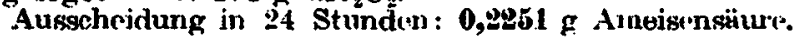

b) Y i s o o h s t ag. Harnmenge: $1800 \mathrm{ccm}$. Gewogen: $0.3544 \mathrm{~g} \mathrm{HgCl}=0,034 ; \mathrm{g} \mathrm{CHF}_{2}()_{2}$.

Ausschoidung in 24 Stunden: $0,213 \mathrm{~g}$ Autrisensaiture.

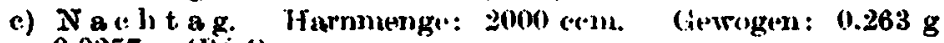
$\mathrm{HgCl}=0.0257 \mathrm{~g}\left(\mathrm{CH}_{2} \mathrm{C}_{2}\right.$.

Aurscheidung in 24 Stumden: 0,181 g Amrisungäure.

Dieses Brgebnis bostätjg t. somit den Befund des ctsten Versuches. Trot\% der großen Zufuhr von $150 \mathrm{~g}$ Traubenzucker and ander(e) Kohlenhydrate war der an Vermohstage und Nachtage gelasse:ne Harn frei vou Zucker.

Die Lintersuchung verschiedener Harnbestandteile ant Ameisengänrebildung dureh hydrolytische Spaltung mit. Phosphorsäure.

Das a u B t r or d e nt l i ch l a ng sa me Ceberdestillieren der Ameisensiiure aus einen Harne, der" mit Phosphorsäure stark angesäuert ist, legt wohl manchem Untersucher, der zum ersten Male diesen Versuch ausfïlut, clie Vermutung nahe, dab Anseisensäure kein präforniertes Stoff dos Harns sei, sondern nus irgendeinem Harnbestandteile durch Hyclrolyse während der Destillation allmählich hervorgehe. In einer älteren Auflage von $H$ o p p e - S e y 1 er und $H$. Th ierfelder's ,Haudbuch der physiologisch- und pathologisch-chemischen Analyse"1) findet sich zuclem die Angabe „Ameisensäure entatehr, bei Zersetzung des Blutfarbstoffes sowie eines, in Harn: häufig a utretenden, kaum gekannten Körers durch Säurenc".

Ich habe nun verschiedene normale und pathologische Bestandteile des menschlichen Harns, mit Phosphorsäure stark angesäuert, in der gleichen Weise der Destillation unterworfen wie den Harn selbst. Beispielsweise wurden aus einer Lösung von $3 \mathrm{~g}$

Sechste Auflage, S. 28 (1893). 
Traubenzucker, $6 \mathrm{~g}$ jtarnstoff, $6 \mathrm{~g}$ Chlornatrium in $300 \mathrm{~g} \mathrm{Wasser}$ unter 7usatz von $30 \mathrm{~cm}$ Phusphorstiure $200 \mathrm{ccm}$ nbdestilliert. Ferner worcle eine 1\% \%ige Milchsilure-. eine 0,2\%ige (Oxalsäure-. eine $1 \%$ ige Kreatinin-, einc $0,05 \%$ ige Harnsüireloisung ... mit wenig Natriumkarbonat bereitet --, jeweils nuch Phosphorsiiurezusntz abdestilliert. Die erhaltenon Destillate wurden in der für die Ameisensäurebestimmung angegebenon WVeise verarbeitor. Bei Milchsiiure, OxRlsäure, Traubenzucker, reinem Fruchtznicker, Harnstoff, Hinpursäure und Kreatinin wurde kein Kalomelniedersshlag erlúlten. wenigstens kein Niederschlas. der in verdünnter Salzsäure unln̈slich geweson wärc. Anffullenderweise lieferte die Harnsiure eine s e h r g e r ing c M e ny a Kalomelnierlerschlag: ein Kontrollversuch verlief gerade so. Ilicwes Ergobnis sei mit allem Vorbehalt angegeben; auf jeden Fall will ich aus demselben. ohne weitere Versuche angestellt \% haben, munächst nicht folgeru, daB Harnsäure, Xanthin oder underc Purinderivate als Ameisensäurebildner für den Tierkürper in Betracht komnien.

\section{Zusammenfassung.}

1. A mejscus i u r a kam als ein nor III a \& e r, ziemlich koustant vorkommender Bcstandteil des menschlichen Harns angesehen werden; die Menge Ameisensïure, die sich in der 24stündigen Harnmonge vorfindet, sehejnt bei verschiedenen Individnen ziemlich stark an schwinken. Bei einigermaßen glejchbleiberider Frnährungsteise, wic dies gegenwärtig hei der wenig Abwechselung bietenden. th Kohlenhylrat reichen, aber an Fett und Eiweiß

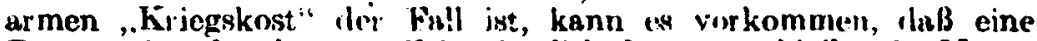
Person eine für längerco 'Jeit viemlich konstant. bleibende Menge Ameisensiumre mit dem L'rin unsscheirlet. So schwankte bei einer erwachsenen männlichen Person der Ameisensïuregehalt ihres Harmes, der von Zeit \&u \%eit bestimmt, wurde, während cines halben .Jahres innerhalh verhältnismüBig kleine: (irenzen. In Harn von \%weinal 24 Stunden fanden sich $0,4 ! 1$ bis $0,(54 \mathrm{~g}$. al so durchsehnittlich $0,562 \mathrm{~g}$ Ameisensäure vor: s.mit betrug die Ansseheidung' für e'inen Tag (t) wn 0,28 g Amejscurvilurc.

2. Zu den Stoffen. die im menschlichen Organismus, wenigstens zum 'Teil, in Amsejsensiure übctgehen. un! die daher cine starke Vermehrung des Ameisensäurcgehaltes des Harns verursachen können, gehört in erstel linie der Methylalkohol. Fine erwachsene mäinnlichr. Persin. die in Verlanfe von acht Tagen $80 \mathrm{~g}$ reinen Methylalkohol in stark verdünnter Lösung eingenommen hatte, hat während dieser \%eit $5,2 \mathrm{~g}$ Ameisensiuure inelur ausgeschieden als unter normalen Verhiiltninsen. Diese Menge Ameinensäure entspricht ungefähr $3 \%$ des eingenonmenen Methylalkohols, die, nach vorauggegangener Oxydation, in Form von Ameisensture mit dem Harn zur Ausscheidung gelangt sind. Dic yrößte Menge der Ameisensïure wurde erst am dritten und vierten Tage nach der Einnalume des Methylalkohols mit dem Harn ausgeschieden. - In toxikologisch or Hinsicht ist beachtenswert, daB der qualitati $\mathbf{r}$ gefúhrte Nachweis der Ameisensäure in einem Harne, bei einer vermuteten Methylnllinholvergif ung, $n$ i $c h$ t 
schon genügt, um auf eine derartige Vergiftung schließen zu können. In aolohen Fillen ist eine quintitative Bestimmung der Aneisens äure des Harns unbed ingt erford er lich. Wemn schon nach Einnahme durchaus. harmloser Mengen von Methylalkohol, z. B. von $30 \mathrm{~g}$ auf zwei Tage verteilt, der Ameisensïuregehalt des flarms stark vermehrt ist, wird dies erst recht der Fall sein, wem es sich um eine akuse Methyl: Wkoholvergiftung mit töllichem Ausgange handelt. In cinem solchen Falle wird ein sehr hoher A no is ens äur egehalt les Harns der betreffenden vergifteten Person von $1 \mathrm{~g}$ und mehr Amejsensäure auf die 24stündige Harnmenge bezogen, selbstverständlich in Verbindung mit anderen Befunden, die Annahme ciner Methylalkoholvergiftung in hohem Grade stützen. - Bemerkenswert ist die aufgefundene Tatsache, daß sich nach Einnahme von Methylalkohol niemals Formaldehyd im Harn nachweisen ließ, wohl aber einmal eine $S$ p 1 r: von unverändert ausgeschiedenem Methylalkohol.

3. A m e is en s ä ure, in Form ihres Natriumsalzes in den Magen eingeführt, wird im menschlichen Körper nur zum Teil verbrannt; bei Einnahme vol $20 \mathrm{~g}$ ameisensaurem Natrium im Verlaufe von zwei Tagen fanden sich 18\% des eingenommenen Formiates im Harne der Versuchsperson vor.

4. Hexamethylentetramin - Urotropin innerlich dargereicht, bewirkte bei einer erwachsenen mämlichen Person keine vermehrte Ameisens äurea $u$ sscheidung. Als an vie: aufeinanderfolgenden Tagen jo $2,5 \mathrm{~g}$, im ganzen also $10 \mathrm{~g}$ i iexamethylentetramin, eingenommen wurden, war der Ameisensüuregehalt des fiarnder Versuchsperson nicht nur nicht vergrößert, sondern nicht unwesentlich erniedrigt. Das Destillat des mit Phosphorsäure stark angesäuert gewesenen flarns enthielt $\mathrm{r}$ e i c h li c h $\mathrm{F}$ or m a ld ehy d. Höchstwahrscheinlich geht Hexamethylentetramin auch als solches in den Harn über. - Die gemachte Beobachtung spricht gegen die Anschauung verschiedener Autoren, claß die Billung der Ameisensäure im Tierkörper aus Trambenzucker. Glycerin u. a. über den Formaldehyd gehe. Wenn diese Annahme für den Nethylalkohol richtig wäre, dann hätt nach Einnahme dieses Alkohols Formaldehyd, der sich gegen Oxydation im menschlichen Körper relativ beständig erwiesen hat. wenigstens in Spuren im Har'n der betreffenden Person auftreten! müssen, was aber bei meinen Versuchen nicht der Fall war.

5. Milch s äu r e, als Natriumsalz per os eingeführt, gehört $n$ i c h $t$ zu den typischen Ameisensäurebildnern im menschlichen Organismus, rlem, als selbst $50 \mathrm{~g}$ milchsaures Natrium im Laufe von zwei Tagen eingenommen wurden, war die normale Ameisensäureausscheidung durch den tiarn nur ganz unwesentlich, und zwar $\mathrm{n}$ ic h t i $m$ m er, vermehts.

6. Per os eingeführter Tr a u b e n z u c k er gehört, nach deu Versuchsergebnissen an zwei erwachsenen Personen, $\mathrm{n}$ ic ht zu den Stoffen, die beim Menschen den Ameisensäuregehalt des Harns beeinflussen können. Obgleich lie eine Person innerhalb ron. 


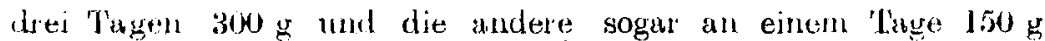
reinen Traubenzicker neben der üblichen Nahrung eingenommen hatte, war der Ameisensäuregehalt des Harns bei beiden Personen an den Versuchstagen und einem Nachtage $n$ ic $h_{t}$ gesteigert. Dieses Frgebnis entspricht ganz der von Pohl ausgesprochenen Anschauung, daß die Kohlenhydrate, Fette und Eiweißstoffe der Nahrung im menschlichen Körper keine Bildung und demnach auch keine gesteigerte Ausscheidung der Ameisensäure durch den Harn hervorrufen können. Anderer * seits steht es im Widerspruche mit den Angaben ron H. D. Daki $n$, J a $n$ ney und $W$ a kem a $n n^{1}$, daß per os oder' intravenös eingefuhrte Glykose sowie andere Kohlenhydrate die Ausfuhr der Ameisensäure stark vermehren. Sehr erheblich ist diese Vermehrung an ausgeschiedener Ameisensäure, wonigstens beim Menschen, allem Ansoheine, nicht gewesen, denn sie hat, manchmal für 24 Stunden nur $35 \mathrm{mg}$ betragen. Die genannten Autoren haben dieses Empfinden wohl selbst gehabt, dem sic schreiben: ,Thes e ef f e c t s (nïmlich Vermehrung der Ameisensảureausscheidung nach reichlicher Zufuhr von Kohlenhydraten) a $r$ e seen.moreclearly in the experimentsondogs." Eine solch geringe "St o iger ung der Ameisensäureausfuhr. von 35-.50 ing Sinire, bezogen anf den 24-Stunden-Harn, liegt aber, meiner Ansicht mach, noch in ner halb der. Sch wan. kungen der normalen Ameisensaureausschei du $\mathrm{ng}$, wie solche auch bei gleichbleibender Ernährungsweise eines Menschen immer rorkommen können.

Aus dem Pharmakologischen Institut der Universität Berlin.

\title{
Ueber den Wirkungswert von Digitalisblättern der ein- und zweijährigen Pflanze.
}

\author{
Von G. Joachimoglu.
}

Nach den Vorschriften des internationalen Uebereinkommens vom 29. November 1906, betreffend die einheitliche Cestaltung der Vorschriften über starkwirkende Arzneimittel, sind in der Ther ppic ausschließlich Digitalisblätter der zweijährigen, blühenden Pflanze zu verwenden. Diese Vorschrift findet sich in den Arzneibüchern aller Kulturstarten, und einige schreiben auch noch vor, daß dic Blätter nicht von der kultivierten, sondern von der wildwachsenden Pflanze zu nehmen sind. Das ist z. B. in den Arzneibüchern Deutschlands, Frankreichs und der Schweiz der Fall. Man könnte vielleicht annehmen, daß die erste Vorschrift bezüglich der Verwendung der

1) The Journ. of Biologioal ('hemistry BA. 14. 35l (1913). 